\title{
Flood Image
}

National Cancer Institute

\section{Source}

National Cancer Institute. Flood Image. NCI Thesaurus. Code C94897.

The image of a flood source. 